\title{
The Light and Heavy Scalars in Unitarized Coupled Channel and Lagrangian Approaches
}

\author{
Frieder Kleefeld \\ Centro de Física das Interacções Fundamentais (CFIF), Instituto Superior Técnico, \\ Edifício Ciência, Piso 3, Av. Rovisco Pais, P-1049-001 LISBOA, Portugal \\ e-mail:kleefeld@cfif.ist.utl.pt
}

\begin{abstract}
Using ideas underlying the flavour-blind "Nijmegen Unitarised Meson Model" (NUMM) [1, 2, 3] we try to understand on the basis of a system of Schrödinger equations with one mesonmeson and one (spinless) quark-antiquark channel coupled by a simple delta-shell transition potential the formation of (e.g. scalar) meson-meson scattering singularities in the complex momentum and energy plane. Surprisingly we are able to describe without direct meson-meson interaction and without any need for glueballs the whole known scalar meson spectrum. "Light" scalar mesons (e.g. $\left.f_{0}(600), \kappa(800), f_{0}(980), a_{0}(985), D_{0}^{*}(2290), \ldots\right)$ are identified to belong to the spectrum of the transition potential, while "heavy" scalars (e.g. $f_{0}(1370), K_{0}^{*}(1430), f_{0}(1500), f_{0}(1710), a_{0}(1450)$, $\left.D_{0}^{*}(2621)(?), D_{0}^{*}(2825)(?), D_{s J}^{*}(2928)(?), \ldots\right)$ are related to the confinement spectrum. Due to the particular value of the charm-strange reduced quark mass level-(anti)crossing in the complex momentum plane [4] occurs which relates the BABAR state $D_{s}(2317)$ [5] to the bare groundstate of the confinement spectrum, while the respective groundstate of the transition potential ends up as $D_{s J}^{*}(2782)$ (?). We conclude with a short comment on (our) recent progress in the consistent quantum field theoretical effective description of resonances within a Lagrangian framework [6, 7, 8].
\end{abstract}

Hadronic excitations with scalar quantum numbers are a topic of heated dispute [9]. Unitarized coupled channel approaches as the one discussed here (see e.g. Ref. [10] and references therein) are particularly useful to understand the non-perturbative formation and nature ${ }^{1}$ of (e.g. scalar) meson-meson scattering singularities in the complex momentum or energy plane, which then may enter as effective degrees of freedom with complex mass and coupling parameters effective Lagrangians describing - according to the "bootstrap" idea - meson-meson scattering already at tree level ${ }^{2}$. For a spherically symmetric situation we couple - in the simplest case - one Schrödinger equation describing a "bound" quark-antiquark system (confining potential $V_{B}(r)$ ) to one Schrödinger equation describing a meson-meson scattering continuum by a transition

\footnotetext{
1 "Heavy" scalars are — disregarding glueballs — mainly associated to the spectrum of the confining quark-antiquark interaction used (e.g. a harmonic oscillator potential), while "light" (dynamically generated [11]) scalars were identified by the author (see e.g. the comments in Refs. [10, 12]) to belong to the spectrum of the meson-(anti)quark transition potential (being to a good approximation of ${ }^{3} P_{0}$-type).

2 The Lagrangian of the Quark-Level Linear Sigma Model (QLL $\sigma$ M) [13, 11, 14] (and references therein) has not only been shown to be an excellent canditate to achieve this task as it reproduces with a minimum of parameters at tree-level a broad spectrum of experimental facts (including the correct prediction of the mass of the now experimentally confirmed $\kappa(800)$-meson) and allowed us to gain some insight in the quark-content of scalar mesons [15], yet could be also "derived" [6] from the Lagrangian of QCD. This "derivation" shows that "glueballs" and "quark-antiquark excitations" seem to be synonymous.
} 
potential denoted by $V_{T}(r)$. I.e., we consider the the following coupled system of radial Schrödinger equations $\left(k:=k_{S}:=\left(2 \mu_{S}\left(E-E_{S}^{(0)}\right)\right)^{1 / 2}, k_{B}:=\left(2 \mu_{B}\left(E-E_{B}^{(0)}\right)\right)^{1 / 2}\right)$ :

$$
\begin{aligned}
& \left(d^{2} / d r^{2}-L(L+1) / r^{2}-2 \mu_{S} V_{S}(r)+k_{S}^{2}\right) \psi_{S}(r)=2 \mu_{S} V_{T}(r) \psi_{B}(r), \\
& \left(d^{2} / d r^{2}-\ell(\ell+1) / r^{2}-2 \mu_{B} V_{B}(r)+k_{B}^{2}\right) \psi_{B}(r)=2 \mu_{B} V_{T}(r) \psi_{S}(r),
\end{aligned}
$$

with $\psi_{S}(0)=0$ and $\psi_{B}(0)=0$. Even though a majority of publications is trying to find the source of "light" scalars in the meson-meson scattering potential $V_{S}(r)$, we are disregarding this interaction in what follows completely (i.e. we set $V_{S}(r)=0$ ). The conveniently normalized ${ }^{3}$ eigensolutions $\phi_{n, \ell}(r)$ of the "bound" system for vanishing transition potential (i.e. $V_{T}(r)=0$ ) correspond to the respective eigenvalues $k_{B, n, \ell}=$ $\left(2 \mu_{B}\left(E_{B, n, \ell}-E_{B}^{(0)}\right)\right)^{1 / 2}$. After integrating the "bound" problem using a Green function ${ }^{4}$ $G_{\ell}\left(r, r^{\prime} ; E-E_{B}^{(0)}\right)$ and reinserting it into the "scattering" problem we arrive at the following generalized scattering problem (with $\left.E=\left(k^{2}+m_{1}^{2}\right)^{1 / 2}+\left(k^{2}+m_{2}^{2}\right)^{1 / 2}\right)$ :

$\left(d^{2} / d r^{2}-L(L+1) / r^{2}+k^{2}\right) \psi_{S}(r)=-2 \mu_{S} \sum_{\ell} V_{T}(r) \int_{0}^{\infty} d r^{\prime} G_{\ell}\left(r, r^{\prime} ; E-E_{B}^{(0)}\right) V_{T}\left(r^{\prime}\right) \psi_{S}\left(r^{\prime}\right)$. Now we will approximate astonishingly well the ${ }^{3} P_{0}$ transition potential by $V_{T}(r)=$ $2 g_{T}\left(2 \mu(E) /\left(2 \mu_{S}\right)\right)^{1 / 2} \delta(r-a)$ with $2 \mu(E)=\partial k^{2} / \partial E=\left(E^{4}-\left(m_{1}^{2}-m_{2}^{2}\right)^{2}\right) /\left(2 E^{3}\right)$ being the relativistic meson-meson phasespace, and hence reduce the generalized scattering problem to a (radial) scattering problem at an effective $\delta$-shell described by the Schrödinger equation $K^{2} \psi_{L}(\rho)=\left(-d^{2} / d \rho^{2}+L(L+1) / \rho^{2}+g \delta(\rho-1)\right) \psi_{L}(\rho)(*)$ with $\rho:=r / a, K:=a k, \psi_{L}(\rho):=\psi_{S, L}(r)$. With $\lambda_{\ell}:=2 g_{T}\left(a G_{\ell}(a, a ; 0)\right)^{1 / 2}$ and $B_{n, \ell}:=\left(a \phi_{n, \ell}(a) \phi_{n, \ell}^{*}(a)\right) /\left(a G_{\ell}(a, a ; 0)\right)$ the dimensionless coupling $g$ displays the structure of the "Resonance Spectrum Expansion" (RSE) [16] of Rupp \& van Beveren:

$$
g=2 \mu(E) \sum_{\ell} \lambda_{\ell}^{2} \sum_{n=0}^{\infty} \frac{B_{n, \ell}}{E-E_{B, n, \ell}} \simeq 2 \mu(E) \sum_{\ell} \lambda_{\ell}^{2}\left(\sum_{n=0}^{N} \frac{B_{n, \ell}}{E-E_{B, n, \ell}}-1\right) .
$$

By construction there holds $\sum_{n=0}^{\infty} B_{n, \ell}\left(E_{B}^{(0)}-E_{B, n, \ell}\right)^{-1}=-1$. The original idea of the RSE [16] was to consider the parameters $B_{n, \ell}, E_{B, n, \ell}, \lambda_{\ell}$ as free parameters to fit selective meson spectra conveniently. Empirically it has become clear [16, 17] that the parameters $\lambda_{\ell}$ and $B_{n, \ell}$ should be considered as "universal" for many different meson spectra provided the product $a \sqrt{\mu_{B}}$ is kept "universal" . In the results displayed below we adopted the philosophy of the NUMM to describe all meson-spectra on the

3 Orthonormality: $\int_{0}^{\infty} d r \phi_{m, \ell}^{*}(r) \phi_{n, \ell}(r)=\delta_{m n}$; completeness: $\sum_{n, \ell} \phi_{n, \ell}(r) \phi_{n, \ell}^{*}\left(r^{\prime}\right)=\delta\left(r-r^{\prime}\right)$.

${ }^{4}$ Green function: $G_{\ell}\left(r, r^{\prime} ; E-E_{B}^{(0)}\right)=-2 \mu_{B} \sum_{n, \ell} \phi_{n, \ell}(r) \phi_{n, \ell}^{*}\left(r^{\prime}\right)\left(k_{B}^{2}-k_{B, n, \ell}^{2}+i \varepsilon\right)^{-1}$.

5 To keep $a \sqrt{\mu_{B}}$ constant became clear from calculations performed in e.g. Ref. [1, 2] based on a transition potential $V_{T}(r)=g \omega \rho_{0}^{-1} \delta\left(r \sqrt{\mu_{B} \omega}-\rho_{0}\right) \bar{V}_{\text {int }}=g\left(\mu_{B} a\right)^{-1} \delta(r-a) \bar{V}_{\text {int }}$ successfully applied to a wide range of vector meson spectra. This transition potential was a simplified version of the harmonic oscillator form of the ${ }^{3} P_{0}$ transition potential inferred by G. Rupp [1] and successfully applied within the NUMM [1, 2, 3] in the representation $\left[V_{T}(r)\right]_{i j}=\tilde{g} \omega c_{i j}\left(E / E_{S}^{(0)}\right)^{1 / 2}\left(r / r_{0}\right) \exp \left(-\left(r / r_{0}\right)^{2} / 2\right)$ with $r_{0}:=\rho_{0}\left(\mu_{B} \omega\right)^{-1 / 2}$. The flavour-blindness [10] of QCD is reflected here not only by the recoupling coefficients $c_{i j}$ (or $\left.\bar{V}_{i n t}\right)\left[1,2\right.$, 3, 18] but also by the "universal" values for $\rho_{0}$ and $\omega\left(\left(\mu_{B} \omega\right)^{-1 / 2}\right.$ has the 
basis of a harmonic oscillator potential ${ }^{6}$ with an "universal" oscillator frequency $\omega=$ $190 \mathrm{MeV}$, and constituent quark masses [14] $m_{u}=m_{d}=337 \mathrm{MeV}, m_{s}=1.44 m_{u}, m_{c} \simeq$ $m_{D}=1865 \mathrm{MeV}$. Hadronic resonances in meson-meson scattering are then determined for Eq. (*) by solving the respective resonance condition $\cot \delta_{L}=i$ with $^{7} \cot \delta_{L}=$ $\left(n_{L}(K) / j_{L}(K)\right)-K /\left(g\left(j_{L}(K)\right)^{2}\right)$. As in Ref. [16] we will choose for the description of scalar mesons $(L=0)$ a RSE with two P-wave $(\ell=1, N=1)$ bare quark-antiquark $\left(q \bar{q}^{\prime}\right)$ states in each meson-meson $\left(M M^{\prime}\right)$ scattering channel. Hence, the respective $\mathrm{RSE}$ resonance condition to be solved in each meson-meson scattering channel is ${ }^{8}$

$$
\frac{2 i K}{1-\exp (2 i K)} \simeq 2 \mu_{M M^{\prime}}^{q \bar{q}^{\prime}}(K) \lambda^{2}\left(\frac{B_{0,1}(\bar{\rho})}{E_{M M^{\prime}}^{q \bar{q}^{\prime}}(K)-E_{B, 0,1}}+\frac{B_{1,1}(\bar{\rho})}{E_{M M^{\prime}}^{q \bar{q}^{\prime}}(K)-E_{B, 0,1}-2 \omega}-1\right) \text {. }
$$

Up to now the bare groundstates of the harmonic oscillator have to be determined empirically. Here we choose ${ }^{9} E_{B, 0,1}=1310 \mathrm{MeV}$ [16] for S-wave $\pi \pi$-, $K K(I=0)$-, $\pi K$-, $\pi \eta_{n \bar{n}}$-scattering, $E_{B, 0,1}=2440 \mathrm{MeV}$ for $S$-wave $D \pi$-scattering, and $E_{B, 0,1}=2545 \mathrm{MeV}$ [17] for S-wave $D K$-scattering. $a_{u \bar{s}}$ and $\bar{\rho}$ are then determined such that for given $\lambda$ the mesons $\kappa(800)$ (pole-position $(714-i 228) \mathrm{MeV}$ [16]) and $f_{0}(980)$ (pole-position $980 \mathrm{MeV})$ are reproduced simultaneously. In a good approximation $f_{0}(980)$ is here assumed to be purely strange [15]. In using $m_{\pi}=140 \mathrm{MeV}$ and $m_{K}=494 \mathrm{MeV}$ we obtain the approximate result $a_{u \bar{s}} \simeq 2.55357 \mathrm{GeV}^{-1}$ and $\bar{\rho} \simeq 1.45555$ yielding immediately $B_{0,1}(\bar{\rho}) \simeq 0.285546 \mathrm{GeV}, B_{1,1}(\bar{\rho}) \simeq 0.0166127 \mathrm{GeV}$, and $\lambda \simeq 1.11572 \mathrm{GeV}^{-1 / 2}$. On the basis of these parameters and $m_{\eta_{n \bar{n}}}=757.9 \mathrm{MeV}$ (for a mixing angle of $41.84^{\circ}$ in $n \bar{n}-s \bar{s}$ basis [19]) we can determine the solution of further selective RSE resonance conditions of interest in choosing e.g. $M, M^{\prime} \in\left\{\pi, \eta_{n \bar{n}}, K, D, \ldots\right\}^{10}$. For S-wave $\pi K-$, $D \pi$-, and $D K$-scattering the results are illustrated graphically in Fig. 1. In all cases there

meaning of an oscillator length). In the RSE $\left(E / E_{S}^{(0)}\right)^{1 / 2}$ finds the interpretation of the square root of the relativistic meson-meson phasespace, as $4 \mu(E) / E_{S}^{(0)}=\left(E^{4}-\left(m_{1}^{2}-m_{2}^{2}\right)^{2}\right) /\left(E^{3}\left(m_{1}+m_{2}\right)\right) \stackrel{m_{1}=m_{2}}{\longrightarrow} E / E_{S}^{(0)}$. ${ }^{6}$ For the harmonic oscillator potential $V_{B}(r)=\frac{1}{2} \mu_{B} \omega^{2} r^{2}$ we have $E_{B, n, \ell}-E_{B}^{(0)}=\omega\left(2 n+\ell+\frac{3}{2}\right)$ and

$$
B_{n, \ell}(\bar{\rho})=\frac{2 \omega \sqrt{\pi}}{\Gamma\left(\frac{1}{2}\left(\ell+\frac{3}{2}\right)\right) \Gamma\left(\frac{1}{2}\left(\ell+\frac{5}{2}\right)\right)} \frac{\left(\bar{\rho}^{2} / 2\right)^{\ell+\frac{1}{2}} e^{-\bar{\rho}^{2}}}{I_{\frac{1}{2}\left(\ell+\frac{1}{2}\right)}\left(\bar{\rho}^{2} / 2\right) K_{\frac{1}{2}\left(\ell+\frac{1}{2}\right)}\left(\bar{\rho}^{2} / 2\right)} \frac{\Gamma\left(\ell+\frac{3}{2}\right) n !}{\Gamma\left(n+\ell+\frac{3}{2}\right)}\left|L_{n}^{\left(\ell+\frac{1}{2}\right)}\left(\bar{\rho}^{2}\right)\right|^{2} .
$$

Here we defined an "universal" parameter $\bar{\rho}:=a\left(\mu_{B} \omega\right)^{1 / 2}$, while $I_{v}(z)$ and $K_{v}(z)$ are modified Bessel functions, and $L_{n}^{(\alpha)}(z)$ are standard generalized Laguerre polynomials.

${ }^{7} \mathrm{~S}$-wave $(L=0)$ meson-meson scattering yields $i=-\cot K-K /\left(g \sin ^{2} K\right) \Leftrightarrow g=2 i K /(1-\exp (2 i K))$. ${ }^{8}$ Here we defined $\lambda:=\lambda_{1}, E_{M M^{\prime}}^{q \bar{q}^{\prime}}(K):=\left(\left(K / a_{q \bar{q}^{\prime}}\right)^{2}+m_{M}^{2}\right)^{1 / 2}+\left(\left(K / a_{q \bar{q}^{\prime}}\right)^{2}+m_{M^{\prime}}^{2}\right)^{1 / 2}$, and $\mu_{M M^{\prime}}^{q \bar{q}^{\prime}}(K):=$ $\left(\left(K / a_{q \bar{q}^{\prime}}\right)^{2}+m_{M}^{2}\right)^{1 / 2}\left(\left(K / a_{q \bar{q}^{\prime}}\right)^{2}+m_{M^{\prime}}^{2}\right)^{1 / 2} / E_{M M^{\prime}}^{q \bar{q}^{\prime}}(K)$.

Note that $a_{n \bar{n}} \sqrt{\mu_{u \bar{u}}}=a_{s \bar{s}} \sqrt{\mu_{s \bar{s}}}=a_{u \bar{s}} \sqrt{\mu_{u \bar{s}}}=a_{c \bar{s}} \sqrt{\mu_{c \bar{s}}}=a_{c \bar{d}} \sqrt{\mu_{c \bar{d}}}$ with $\mu_{q \bar{q}^{\prime}}:=m_{q} m_{\bar{q}^{\prime}} /\left(m_{q}+m_{\bar{q}^{\prime}}\right)$.

${ }^{9}$ Note that $k \simeq 766 \mathrm{MeV} \simeq 4 \omega$ resulting from $E_{B, 1,1}$ seems to be to a good approximation "universal".

${ }^{10}$ For S-wave $\pi \pi-, K K(I=0)-, \pi K-, \pi \eta_{n \bar{n}^{-}}, D \pi$-, and $D K$-scattering we find the following pole positions in the complex energy plane: $\pi \pi$-scattering: $(516-i 412) \mathrm{MeV}\left(f_{0}(600)\right),(1385-i 81) \mathrm{MeV}$ $\left(f_{0}(1370), E_{0,1}^{\text {dressed }}\right),(1694-i 4) \mathrm{MeV}\left(f_{0}(1710), E_{1,1}^{\text {dressed }}\right) ; K K(I=0)$-scattering: $980 \mathrm{MeV}\left(f_{0}(980)\right)$, $351 \mathrm{MeV}$ (Virtual BS), $(1452-i 191) \mathrm{MeV}\left(f_{0}(1500), E_{0,1}^{\text {dressed }}\right),(1692-i 11) \mathrm{MeV}\left(f_{0}(1710), E_{1,1}^{\text {dressed }}\right)$;

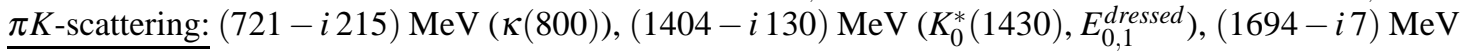



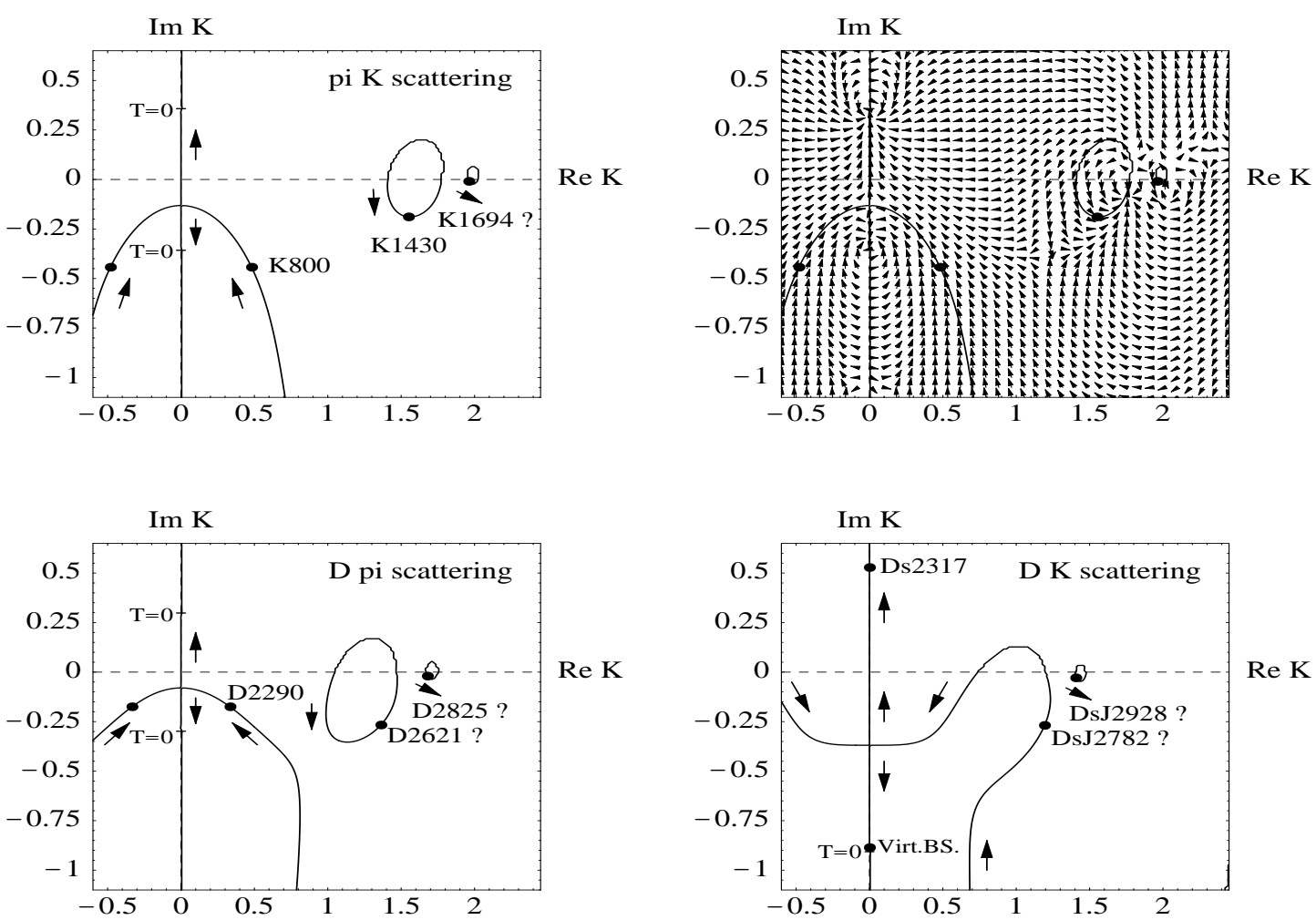

FIGURE 1. Propagation of poles for increasing $\lambda^{2}$ in the complex $K$-plane. Solid lines: curves with $\operatorname{Im}\left[\lambda^{2}\right]=0$. " $T=0$ " indicates a zero of the amplitude due to vanishing phasespace ( $\simeq$ "Adler-zero"). Resonances at $\lambda \simeq 1.11572 \mathrm{GeV}^{-1 / 2}$ are denoted by $\bullet$. S-wave scattering: $\pi K: \kappa(800), K_{0}^{*}(1430)$, $K_{0}^{*}(1694)\left(\right.$ ?); $\underline{D \pi}: D_{0}^{*}(2290), D_{0}^{*}(2621)(?), D_{0}^{*}(2825)(?) ; \underline{D K}: D_{s}(2317), D_{s J}^{*}(2782)(?), D_{s J}^{*}(2928)(?)$.

starts for $\lambda=0(\Rightarrow g=0)$ a $\delta$-shell pole trajectory at $K=\pi / 2-i \infty$. For $g \rightarrow \infty$ this pole should behave like a particle in a box and end up at $K \rightarrow \pi$. Instead, it collides for increasing $\lambda$ with the pole stemming from the bare groundstate of the confinement problem and gets deflected either to the "left" $(\pi K, D \pi)$ or to the "right" $(D K)^{11}$.

$\left(E_{1,1}^{\text {dressed }}\right) ; \pi \eta_{n \bar{n}}$-scattering: $(960-i 107) \mathrm{MeV}\left(a_{0}(985)\right),(1423-i 161) \mathrm{MeV}\left(a_{0}(1450), E_{0,1}^{\text {dressed }}\right)$, $(1693-i 8) \mathrm{MeV}\left(E_{1,1}^{\text {dressed }}\right) ;$ i $\pi$-scattering: $(2073-i 70) \mathrm{MeV}\left(D_{0}^{*}(2290)\right),(2621-i 163) \mathrm{MeV}\left(E_{0,1}^{\text {dressed }}\right)$, $(2825-i 13) \mathrm{MeV}\left(E_{1,1}^{\text {dressed }}\right)$; $D K$-scattering: $(2782-i 166) \mathrm{MeV}\left(D_{s J}^{*}(? ? ?)\right), 2244 \mathrm{MeV}\left(D_{s}(2317)\right.$, $\left.E_{0,1}^{\text {dressed }}\right), 1907 \mathrm{MeV}$ (Virtual BS, $\left.E_{0,1}^{\text {dressed }}\right),(2928-i 20) \mathrm{MeV}\left(E_{1,1}^{\text {dressed }}\right)$.

${ }^{11}$ Consequently, the origin of "light" scalar mesons, the strong distortions of the groundstates of the "observed" confinement spectrum, and the absence of "light" non-scalar mesons for realistic transition potentials (due to the centrifugal barrier) get a nice explanation. The flavour content for $f_{0}(600), f_{0}(980)$, $f_{0}(1370), f_{0}(1500)$ is well consistent with Ref. [15], while observed pole-positions correspond nicely to values obtained for a realistic transition potential [3]. The too large imaginary part of the $f_{0}(600)$ pole is an artefact of the used one-channel model. Furthermore, we observe a twofold nature of the $f_{0}(1710)$ being at the same time $n \bar{n}$ (relation to $f_{0}(600)$ ) and $s \bar{s}$ (relation to $f_{0}(980)$ ). The BABAR state $D_{s}(2317)$ [5] and BELLE state $D_{0}^{*}(2290)$ [20] are reproduced, while the respective next higherlying states are predicted to 
Shortly we address effective Lagrangian approaches: scalar mesons are characterized within a Lagrangian close to bootstrap typically by complex mass and coupling parameters to be determined by a coupled channel approach. The formalism to describe fields within a non-Hermitian Lagrangian has been provided [6, 7, 8]. The QCD-Lagrangian has been mapped [6] into a QLL $\sigma$ M-Lagrangian replacing the gluon-quark interaction $g \overline{q_{+}^{c}}(x) \not A(x) q_{-}(x)$ by a non-Hermitian meson-quark interaction. The resulting scalarmeson-quark interaction $i \sqrt{2 N_{F} / N_{c}} g \overline{q_{+}^{c}}(x) S(x) q_{-}(x)$ yields an asymptotic free theory which is $P T$-symmetric [21] admitting a real spectrum and a probability interpretation.

\section{ACKNOWLEDGMENTS}

This work has been supported by the Fundação para a Ciência e a Tecnologia of the Ministério da Ciência e da Tecnologia (e do Ensinio Superior) of Portugal, under Grants no. PRAXIS XXI/BPD/20186/99, SFRH/BDP/9480/2002, POCTI/FNU/49555/2002.

\section{REFERENCES}

1. G. Rupp, Doctoral Thesis (Catholic University of Nijmegen, 1982).

2. E. van Beveren et al., Phys. Rev. D 21 (1980) 772; D 22 (1980) 787; Phys. Rev. D 27 (1983) 1527; J. E. Ribeiro, Phys. Rev. D 25 (1982) 2406.

3. E. van Beveren et al., Z. Phys. C 30 (1986) 615.

4. E. Hernández et al., Rev. Mex. Fis. 49 (S4) (2003) 17; Phys. Rev. A 67 (2003) 022721.

5. B. Aubert et al. [BABAR Collaboration], Phys. Rev. Lett. 90 (2003) 242001.

6. F. Kleefeld, AIP Conf. Proc. 660 (2003) 325 |hep-ph/0211460|.

7. F. Kleefeld, hep-th/0310204 Few-Body Systems Suppl. 15 (2003) 201; Acta Phys. Polon. B 30 (1999) 981; Vol. I of Proc. XIV ISHEPP 98, 17-22.8.1998, Dubna, Russia, pp. 69-77 (Eds. A.M. Baldin, V.V. Burov) |nucl-th/9811032|; F. Kleefeld, Doctoral Thesis (University of Erlangen-Nürnberg, 1999).

8. F. Kleefeld, E. van Beveren and G. Rupp, Nucl. Phys. A 694 (2001) 470.

9. S. F. Tuan, hep-ph/0303248, D. V. Bugg, Phys. Lett. B 572 (2003) 1.

10. E. van Beveren et al., AIP Conf. Proc. 660 (2003) 353 [hep-ph/0211411|.

11. E. van Beveren, F. Kleefeld, G. Rupp, M. D. Scadron, Mod. Phys. Lett. A 17 (2002) 1673.

12. E. van Beveren, G. Rupp, hep-ph/0306185

13. R. Delbourgo, M. D. Scadron, Mod. Phys. Lett. A 10 (1995) 251; Int. J. Mod. Phys. A 13 (1998) 657; M. D. Scadron et al., Nucl. Phys. A 724 (2003) 391; AIP Conf. Proc. 660 (2003) 311.

14. M. D. Scadron, G. Rupp, F. Kleefeld, E. van Beveren, hep-ph/0309109 hep-ph/0307003

15. F. Kleefeld, E. van Beveren, G. Rupp, M. D. Scadron, Phys. Rev. D 66 (2002) 034007.

16. E. van Beveren, G. Rupp, Eur. Phys. J. C 22 (2001) 493.

17. E. van Beveren, G. Rupp, Phys. Rev. Lett. 91 (2003) 012003; hep-ph/0306051 hep-ph/0306155

18. E. van Beveren, G. Rupp, Eur. Phys. J. C 11 (1999) 717; Phys. Lett. B 454 (1999) 165.

19. D. Kekez, D. Klabučar, M. D. Scadron, J. Phys. G 27 (2001) 1775.

20. P. Krokovny [BELLE Collaboration], hep-ex/0210037

21. C. M. Bender et al., hep-th/0303005, M. Znojil, quant-ph/0309100

be $D_{s J}^{*}(2782)(?)$ and $D_{0}^{*}(2621)(?)$. Note also the prediction of $K_{0}^{*}(1694)(?)$ and $a_{0}(1693 \ldots 1694)(?)$. 